\title{
ITERATIVE CONSTRUCTION OF FIXED POINTS OF A DISSIPATIVE TYPE OPERATOR
}

\section{XINLONG WENG}

Let $D$ be a nonempty closed convex subset of a Hilbert space, $H$, with inner product $\langle\cdot, \cdot\rangle$, and let $T: D \mapsto D$ satisfy the dissipative type condition

$$
\operatorname{Re}\langle T(x)-T(y), x-y\rangle \leq C\|x-y\|^{2}
$$

for some $C<1$ and all $x, y \in D$. If $T$ is also Lipschitz continuous on $D$, then $T$ has precisely one fixed point $\bar{x} \in D[1]$. Moreover, if $\left\{C_{n}\right\} \subset(0,1]$ satisfies the following conditions:

$$
\lim _{n \rightarrow \infty} C_{n}=0 \quad \sum_{n=0}^{\infty} C_{n}=\infty
$$

then the recursion

$$
x_{n+1}=\left(1-C_{n}\right) x_{n}+C_{n} T\left(x_{n}\right) \quad x_{0} \in D
$$

will converge to $\bar{x}$ [2]. Rhoades [3] has shown that if $D$ is a bounded interval of $R^{1}$ and $T$ maps $D$ into $D$, then $\left\{x_{n}\right\}$ will converge to some fixed point of $T$ if $T$ is merely continuous and $\left\{C_{n}\right\}$ satisfies (2). The process (3) and the more general processes of Mann [4] and Ishikawa [5] have been investigated for operators satisfing various conditions of the continuity and contractivity type on special Banach spaces, a summary of some contributions to this field is given in $[3,6-8]$. 
In [16], Dunn introduced the weaker version of (1) namely,

$$
\operatorname{Re}\langle\xi-\bar{x}, x-\bar{x}\rangle \leq C\|x-\bar{x}\|^{2}
$$

for some $\bar{x} \in D, C<1$, and for all $x \in D, \xi \in T x$. Here $T$ is set valized and no continuity restrictions of any kind are imposed on $T$, also $D$ need not be closed ur open. Instead, it is assumed that the range of $T$ is bounded. Dunn showed that if $x \in T(x)$, i.e., if $x$ is a fixed point of $T$ then $x=\bar{x}$, so that $T$ can have at most one fixed point. Moreover, if $\left\{x_{n}\right\}$ is a sequence in $D$ satisfying

$$
x_{n+1}=\left(1-C_{n}\right) x_{n}+C_{n} \xi_{n}
$$

where $\xi_{n} \in T\left(x_{n}\right)$, with $\left\{C_{n}\right\} \subset(0,1]$ satisfying

$$
\sum_{n=0}^{\infty} C_{n}=\infty \quad \sum_{n=0}^{\infty} C_{n}^{2}<\infty
$$

then $\left\{x_{n}\right\}$ strongly converges to $\bar{x}$.

Recently, Chidume [17] exiended the results of Dunn [16] from Hilbert spaces to the $L_{p}$ spaces for $p \geq 2$, which has at least two disjoint sets of positive finte measure.

Our purpose in this paper is to extend all results of Chidume and Dunn to the general complex uniformly smooth Banach spaces and obtain a convergence rate in the $L^{p}, e^{p}, W_{m}^{p}$ spaces for $1<p<\infty$.

\section{Definitions and Preliminary Results}

Let $X$ be an arbitrary Panach space with dim $X \geq 2$, the moduhs of convexty $\delta_{X}(\epsilon), 0<\epsilon \leq 2$, of $X$ is defined by

$$
\delta_{X}(\epsilon)=\inf \{1-\|x+y\| / 2: x, y \in X,\|x\|=\|y\|=1,\|x-y\|=\epsilon\}
$$

$X$ is said to be uniformly convex if $\delta_{X}(\epsilon)>0$ for every $\epsilon>0$, and uniformly smooth if the dual space $X^{*}$ is uniformly convex. The estimation of the modulus of convexity for the spaces $L^{p}, e^{p}, W_{m}^{p}, 1<p<\infty$ are [21]:

$$
\begin{array}{ll}
\delta_{X}(\epsilon) \geq(p-1 / 16) \epsilon^{2}, & 1<p \leq 2 \\
\delta_{X}(\epsilon) \geq p^{-1}(\epsilon / 2)^{p}, & p \geq 2
\end{array}
$$


For the Banach space $X$, we shall denote by $J$ the normalized duality mapping from $X$ to $2^{X \text { "* }}$ given by

$$
J_{x}=\left\{f^{*} \in X^{*}:\left\|f^{*}\right\|^{2}=\|x\|^{2}=\left\{x, f^{*}\right\rangle\right\}
$$

where $\langle\cdot$,$\rangle denotes the generalized duality pairing. If X$ is uniformly smooth, then $J$ is single-valued and is uniformly contirizous on bounded set.

We derine for positive $t$

$$
\beta(t)=\sup \left\{\left(\|x+t y\|^{2}-\|x\|^{2}\right) / t-2 \operatorname{Re}\langle y, J(x)\rangle:\|x\| \leq 1,\|y\| \leq 1\right\}
$$

Clearly $\beta:(0, \infty) \rightarrow[0, \infty)$ is rondecreasing, continuous and $\beta(c t) \leq c \beta(t)$ for $c \geq 1$. Also we have

Lemma 1. (Refer [18]) If $X$ is a uniformly smooth Banach space and $\beta(t)$ is defined as above, then $\lim _{t \rightarrow 0+} \beta(t)=0$ and

$$
\|x+y\|^{2} \leq\|x\|^{2}+2 \operatorname{Re}\langle y, J(x)\rangle+\max \{\|x\|, 1\}\|y\| \beta(\|y\|)
$$

for all $x, y \in X$.

Proof. The proof is same as S.Reich proved for a real uniformly smooth Banach space [18].

Lemmer 2. Let $\beta_{n}$ be a noneguive serience satisfying

$$
\beta_{n+1} \leq\left(1-\delta_{n}\right) \beta_{n}+\sigma_{n}
$$

with $\delta_{n} \in[0,1], \sum_{i=1}^{\infty} \delta_{i}=\infty$ and $\sigma_{n}=o\left(\delta_{n}\right)$. Then $\lim _{n \rightarrow \infty} \beta_{n}=0$.

Proof. Since $\sigma_{n}=o\left(\delta_{n}\right)$, let $\sigma_{n}=\epsilon_{n} \cdot \delta_{n}$, and $\epsilon_{n} \rightarrow 0$. By a straightforward induction, one obtains

$$
0 \leq \beta_{n+1} \leq \Pi_{j=k}^{n}\left(1-\delta_{j}\right) \beta_{k}+\sum_{j=k}^{n}\left[\delta_{j} \prod_{i=j+1}^{n}\left(1-\delta_{i}\right)\right] \epsilon_{j}
$$


We have

$$
\prod_{j=k}^{n}\left(1-\delta_{j}\right) \leq e^{-\sum_{j=k}^{n} \delta_{j}} \rightarrow 0
$$

and.

$$
\sum_{j=k}^{n} \delta_{j} \prod_{i=j+1}^{n}\left(1-\delta_{i}\right) \leq 1 \quad \forall n, k
$$

Given $\epsilon>0$, pick $k$ such that $\epsilon_{j} \leq \epsilon$ for all $j \geq k$, from (*) we have

$$
0 \leq \liminf \beta_{n} \leq \limsup \beta_{n} \leq \epsilon
$$

Letting $\epsilon \rightarrow 0$, we obtain $\lim _{n \rightarrow \infty} \beta_{n}=0$.

For the uniformly smooth Banach spaces, we consider a mapping $T: D \mapsto 2^{D}$ satisfying

$$
\operatorname{Re}\langle\xi-\bar{x}, J(x-\bar{x})\rangle \leq C\|x-\bar{x}\|^{2}
$$

for some $\bar{x} \in D, C<1$, and for all $x \in D, \xi \in T(x)$. Actually, (8) is a natural generalization to a Banach space of the (4) for a Hilbert space.

\section{Main Results}

Theorem 1. Let $D$ be a subset of a uniformly smooth Banach space and $T: D \mapsto 2^{D}, \bar{x} \in D$ satisfy (8). If $x$ is a fixed point of $T$, then $x=\bar{x}$, thus $T$ can have at most one fixed point.

Moreover suppose the range of $T$ is bounded and let $\left\{x_{n}\right\} \subset D$ be generated by (5) with $\left\{C_{n}\right\} \subset(0,1]$ satisfying $(2)$ then $\left\{x_{n}\right\}$ strongly converges to $\bar{x}$.

Proof. Let $x$ be a fixed point of $T$, i.e., $x \in T(x)$. The condition (8) yields

$$
\langle x-\bar{x}, J(x-\bar{x})\rangle \leq C\|x-\bar{x}\|^{2}
$$

so that

$$
\|x-\bar{x}\|^{2} \leq C\|x-\bar{x}\|^{2}
$$

Since $C<1$, this gives $x-\bar{x}$. 
Now set

$$
\beta_{n}=\left\|x_{n}-\bar{x}\right\|^{2}
$$

and

$$
d=\sup \{\|\xi-\bar{x}\|: \xi \in T(x), x \in D\}
$$

Because $C_{n} \rightarrow 0$, it is easy to show there exists an integer $N \geq 1$ such that when $n \geq N$

$$
\left[1-(1-C) C_{n}\right]^{2}+d^{2} C_{n} \beta\left(C_{n}\right) \leq 1
$$

Let $B=\max \left\{\beta_{i}: 1 \leq i \leq N, 1\right\}$. First we want to show $\beta_{n} \leq B^{2}$ and

$$
\beta_{n+1} \leq\left[1-(1-C) C_{n}\right]^{2} \beta_{n}+B^{2} d^{2} C_{n} \beta\left(C_{n}\right) .
$$

From (5), (7), (8) and (9), we have

$$
\begin{aligned}
\beta_{n+1}= & \left\|x_{n+1}-\bar{x}\right\|^{2}=\left\|\left(1-C_{n}\right)\left(x_{n}-\bar{x}\right)+C_{n}\left(\xi_{n}-\bar{x}\right)\right\|^{2} \\
\leq & \left(1-C_{n}\right)^{2}\left\|x_{n}-\bar{x}\right\|^{2}+2 C_{n}\left(1-C_{n}\right) \operatorname{Re}\left\langle\xi_{n}-\bar{x}, J\left(x_{n}-\bar{x}\right)\right\rangle \\
& +\max \left\{\left\|x_{n}-\bar{x}\right\|, 1\right\} C_{n}\left\|\xi_{n}-\bar{x}\right\| \beta\left(C_{n}\left\|\xi_{n}-\bar{x}\right\|\right) \\
\leq & \left(1-C_{n}\right)^{2} \beta_{n}+2 C_{n}\left(1-C_{n}\right) C \beta_{n}+\max \left\{\left\|x_{n}-\bar{x}\right\|, 1\right\} d^{2} C_{n} \beta\left(C_{n}\right) \\
\leq & {\left[1-(1-C) C_{n}\right]^{2} \beta_{n}+\max \left\{\beta_{n}, 1\right\} d^{2} C_{n} \beta\left(C_{n}\right) }
\end{aligned}
$$

For $n \leq N$, by the definition of number $B$, we have $\beta_{n} \leq B^{2}$. For $n \geq N$, we apply induciton: assume $\beta_{n} \leq B^{2}$ then

$$
\begin{aligned}
\beta_{n+1} & \leq\left[1-(1-C) C_{n}\right]^{2} \beta_{n}+B^{2} d^{2} C_{n} \beta\left(C_{n}\right) \\
& \leq\left\{\left[1-(1-C) C_{n}\right]^{2}+d^{2} C_{n} \beta\left(C_{n}\right)\right\} B^{2} \\
& \leq B^{2}
\end{aligned}
$$

So we obtain $\beta_{n} \leq B^{2}$ for all $n$ and from (11) we get (10). Now apply Lemma 2 to inequality (10). We get that $\left\{x_{n}\right\}$ strongly converges to the fixed point $\bar{x}$.

Remark 1. If $T x \neq \emptyset$ for $x \in D$, then by the axiom of choice $T$ has a single-valued section, $T^{*}: D \mapsto D, T^{*} x \in T x$. For any such section, and for $D$ convex and $\left\{C_{n}\right\} \subset(0,1]$, the recursion

$$
x_{n+1}=\left(1-C_{n}\right) x_{n}+C_{n} T^{*} x_{n} \quad x_{0} \in D
$$


generates a sequence $\left\{x_{n}\right\} \subset D$.

Remark 2. Theorem 1 is a generalization of [17] in Banach spaces, and for $\left\{C_{n}\right\}$ we use weaker conditions (2) to replace stronger conditions (6) that Chidume used in $L_{p}(p \geq 2)$ spaces.

For the special uniformly smooth Banach spaces $X=L^{p}, e^{p}, W_{m}^{p}, 1<p<$ $\infty$, we have the estimate [19]:

$$
\beta(t) \leq M t^{s-1}
$$

Where $s=2$ if $2 \leq p<\infty, s=p$ if $1<p<2$, and $M$ is some constant. Then we are able to obtain a convergence rate in the setting of Theorem 1 .

Theorem 2. Let $X=L^{p}, e^{p}, W_{m}^{p}$ and $T,\left\{x_{n}\right\}, C_{n}$ be as in Theorem 1. Then we can find $\left\{C_{n}^{*}\right\}$ such that for corresponding sequence $\left\{x_{n}^{*}\right\}$ we have the estimate

$$
\left\|x_{n}^{*}-\bar{x}\right\| \leq O\left(1 / n^{(s-1) / 2}\right) .
$$

Where $s=2$ if $2 \leq p<\infty, s=p$ if $1<p<2$. In a sense to be made precise below, the above estimate is the "best estimate" and the sequence $\left\{x_{n}^{*}\right\}$ is the "best sequence" in this class for each fixed value of the constant $C$ in (8).

Proof. Let

$$
\begin{aligned}
C_{n}^{*} & =\frac{(1-C)^{1 /(s-1)}}{1+n(1-C)^{s /(s-1)}} \\
x_{n+1}^{*} & =\left(1-C_{n}\right) x_{n}^{*}+C_{n} T x_{n}^{*} \\
\beta_{n}^{*} & =\left\|x_{n}^{*}-\bar{x}\right\|^{2} \\
\alpha_{n+1}^{*} & =\left[1-(1-C) C_{n}^{*}\right]^{s} \alpha_{n}^{*}+C_{n}^{* s} \quad \alpha_{1}^{*} \geq\left(M B^{* 2} d^{2}\right)^{-1} \beta_{1}^{*}
\end{aligned}
$$

Solve above equations we get

$$
\alpha_{n}^{*}=\frac{\alpha_{1}^{*}}{\left[1+(n-1) \alpha_{1}^{* 1 /(s-1)}(1-C)^{s /(s-1)}\right]^{s-1}} .
$$

From (10) and the estimation of $\beta(t)$ in $L_{p}$ spaces, we have $\beta_{n}^{*} \leq\left(M B^{* 2} d^{2}\right) \alpha_{n}$, $n=1,2, \ldots$ Thus, we get (12). Now we turn to prove following claim. Claim: 
for any sequence $\left\{C_{n}\right\}$, we get sequence

$$
\alpha_{n+1}=\left[1-(1-C) C_{n}\right]^{s} \alpha_{n}+C_{n} \quad \alpha_{1}=\alpha_{1}^{*}
$$

Then we have $\alpha_{n}^{*} \leq \alpha_{n}, n=1,2,3 \ldots$ Consider the function

$$
g(x)=[1-(1-C) x]^{s} \alpha_{n}^{*}+x^{s}
$$

Then

$$
g^{\prime}(x)=-(1-C) s(1-(1-C) x)^{s-1} \alpha_{n}^{*}+s x^{s-1}
$$

Let $g^{\prime}(x)=0$, we get

$$
x=\frac{\alpha_{n}^{* 1 /(s-1)}(1-C)^{1 /(s-1)}}{1+\alpha_{n}^{* 1 /(s-1)}(1-C)^{s /(s-1)}} .
$$

Combine this equation with previous equation, we have $x=C_{n}^{*}$, i.e. the minimum point is $C_{n}^{*}$. We apply induction: if $\alpha_{k}^{*} \leq \alpha_{k}$ then

$$
\begin{aligned}
\alpha_{k+1}^{*} & =\left[1-(1-C) C_{k}^{*}\right]^{s} \alpha_{k}^{*}+C_{k}^{* s} \\
& \leq\left[1-(1-C) C_{k}\right]^{s} \alpha_{k}^{*}+C_{k}^{s} \\
& \leq\left[1-(1-C) C_{k}\right]^{s} \alpha_{k}+C_{k}^{s}=\alpha_{k+1}
\end{aligned}
$$

The proof is completed.

\section{References}

[1] F. E. Browder and W. V. Petryshyn, "Construction of fixed points of nonlinear mappings in Hilbert space", J. Math. Anal. Appl. 20 (1967), 197-228

[2] J. C. Dunn, "On recursive averaging processes and Hilbert space extensions of the contraction mapping principle", J. Franklin Inst 295 (1973), 117-133

[3] B. E. Rhoades, "Fixed point iterations using infinite matrices", Trans. Amer. Math. Soc. 196 (1974), 161-176

[4] W. R. Mann, "Mean value methods in iteration", Proc. Amer. Math. Soc. 4 (1953), 506-510

[5] S. Ishikawa, "Fixed points by a new iteration method", Proc. Amer. Math. Soc. 44 (1974), 14.7-150

[6] B. E. Rhoades, "Fixed point iterations using infinite matrices, II", in Constructuve and Computational Methods for Differential and Integral Equations, Vol. 430, pp. 390-395 (1974) 
[7] B. E. Rhoades, "Fixed point iterations using infinite matrices, III", in Proceedings of the Conference on Computing Fixed Points with Applications, (1974)

[8] B. E. Rhoades, "Comments on two fixed point iteration methods", J. Math. Anal. Appl., (1975)

[9] H. D. Block, "Estimates of error for two modifications of the Robbins-Monro stochastic approximation process", Ann. Math. Statist. 28 (1957), 1003-1010

[10] R. E. Bruck, Jr., "The iterative solution of the equation $y \in x+T x$ for a monotone operator T in Hilbert space", Bull. Amer. Math. Soc. 79 (1973), 1258-1261

[11] R. E. Bruck, Jr., "A strongly convergent iterative solution of $0 \in U(x)$ for a maximal monotone operator $U$ in Hilbert space", J. Math. Anal Appl. 48 (1974), 114-126

[12] R. E. Burck, Jr., "An iterative solution of a variational inequality for certain monotone operators in Hilbert space", Bull. Amer. Math. Soc. 81 (1975), 890-892

[13] V.F. Demyanov and A. M. Rulbinov, "Approximate Methods in Optimization Problems", American Elsevier, New York, 1970

[14] E. G. Gilbert, "An iterative procedure for computing the minimum of a quadratic form on a convex set", J. SIAM Contral 4 (1966), 61-80

[15] R. T. Rockafellar, "Convex Analysis", Princeton Univ. Press, Princeton N. J., 1970

[16] J. C. Dunn, "Iterative construction of fixed points for multivalued operators of the monotone type", Journal of Functional Analysis 27 (1978), 38-50

[17] C. E. Chidume, "Iterative construction of fixed points for multivalued operators of the monotone type", Applicable Analysis 23 (1986) 209-218

[18] S. Reich, "An iterative procedure for constructing zeros of accretive sets in Banach spaces", Journal of Nonlinear Analysis 2 (1978), 85-92

[19] S. Reich, "Constructive techniques for accretive and monotone operators", Applied Nonlinear Analysis, Arlington, TX (1979)

[20] T. Kato, "Nonlinear semigroups and evolution equations", J. Math. Soc. Japan 19 No.4, (1967) 509-519

[21] Ya. I. A1'ber and A. I. Notik, "Geometric properties of Banach spaces and approximate methods for solving nonlinear operator equations Soviet Math", Dokl. 29 No.3 (1984) 611-615

Department of Mathematics, Marshall University, Huntington, West Virginia 25755, U.S.A. 